\title{
Vegetación de Sierra Rica: el papel de la biogeografía en la formación de las comunidades vegetales en Manuel Benavides, Chihuahua, México
}

\author{
Vegetation of Sierra Rica: the role of biogeography in the formation \\ of plant communities in Manuel Benavides, Chihuahua, Mexico
}

\begin{abstract}
Alicia Juárez Pérez', Alicia Melgoza², Jesús A. Fernández²*
Juárez Pérez, A., Melgoza, A., Fernández, J. A. Vegetación de Sierra Rica: el papel de la biogeografía en la formación de las comunidades vegetales en Manuel Benavides, Chihuahua, México. Investigación y Ciencia de la Universidad Autónoma de Aguascalientes. Número 70: 84-91, eneroabril 2017.
\end{abstract}

\section{RESUMEN}

Sierra Rica pertenece al Área de Protección de flora y fauna Cañón de Santa Elena, en el municipio de Manuel Benavides, Chihuahua, México y se considera una isla del cielo biogeográfico. Aquí se presenta una caracterización biogeográfica del área que se pretende ayude a entender la magnitud de su diversidad vegetal y sus patrones de distribución. Se realizó una revisión bibliográfíca complementada con visitas al área de estudio. El origen geológico fue durante la actividad volcánica presente en la era Cenozoica que dio lugar a factores físicos importantes para la distribución de la vegetación. Entre estos factores se identifican microclimas, aspectos fisiográficos y edafológicos, y entre los aspectos bióticos, fuerzas evolutivas como la selección, microorganismos del suelo, mecanismos de dispersión e interacciones bióticas. El establecimiento del bosque de pino, del bosque de encino, pastizal y matorral ha sufrido cambios a través del tiempo; sin embargo, hay elementos que no han cambiado en composición, sólo en distribución.

Palabras clave: biogeografía, Desierto Chihuahuense, distribución geográfica, fisiografía, isla de montaña, tipos de vegetación.

Keywords: biogeography, Chihuahua Desert, geographic distribution, physiography, sky island, vegetation types.

\section{Recibido: 13 de octubre de 2015, aceptado: 19 de agosto de 2016}

Maestría en Producción Animal y Recursos Naturales, Facultad de Zootecnia y Ecología, Universidad Autónoma de Chihuahua, México.

2 Departamento de Recursos Naturales, Facultad de Zootecnia y Ecolo-

gía, Universidad Autónoma de Chihuahua, México.

* Autor para correspondencia: afernandezf@uach.mx
ABSTRACT

The Sierra Rica belongs to the Flora and Fauna Protection Area Santa Elena Canyon, and it is placed in the municipality of Manuel Benavides of Chihuahua, Mexico, and is considered a biogeographic sky island. Here we present for the first time, a biogeographic characterization of this area, providing a review that will help to understand plant diversity, its origins and distributional patterns. We made a bibliographic review complemented with fieldwork performed at the study area. The geological origin of Sierra Rica goes back to the volcanic activity of the Cenozoic era, giving rise to abiotic and biotic features relevant to plant distribution. Among those factors we identified microclimates, soil and physiographic features, as well as evolutionary forces like selection, soil microorganisms, dispersal mechanisms, and organismic interactions. The establishment of the pine and oak forests, grassland and shrub land communities have suffered changes through time, however, there are elements that did not change composition, only change distribution.

INTRODUCCIÓN

La mayor parte del estado de Chihuahua se ubica en el Desierto Chihuahuense (DC), con ecosistemas de pastizal, matorral y bosque de coníferas (CONABIO, 2014). Dentro de los ecosistemas que ocupan las planicies del DC se presentan montañas aisladas de elevada altitud llamadas islas del cielo (McCormack et al., 2009; Granados Sánchez et al., 2011). Estas zonas de montaña destacan por su vegetación y fisiografía diferente al de las áreas que les circundan, pero también por ser excelentes sistemas para investigar de qué forma, fuerzas evolutivas como la selección natural y la deriva genética actúan para generar diversidad biológica. 


\section{IIVESTIGAGIÓn Y CUERCIA DE LA UחIVERSIDAD AUTÓNOMA DE AGUASCALIERTES}

Sierra Rica es parte de un sistema de islas del cielo llamado Archipiélago de la Sierra Madre, que incluye montañas del suroeste de Estados Unidos y del norte de México (McCormack et al., 2009). Sierra Rica es una montaña aislada que presenta vegetación de bosque, matorral y pastizal rodeada de valles con vegetación de matorral desértico que impide el intercambio genético con poblaciones que habitan otras islas del cielo con vegetación similar. Para entender la dimensión de los patrones de distribución heterogéneos que se presentan, la biogeografía es una herramienta que permite analizar y estudiar los factores causantes de la distribución de especies a través del tiempo ecológico e histórico (Cox y Moore, 1993; Lomolino et al., 2010). Estos factores son de carácter biótico, como preferencias de hábitat o características genéticas y abióticos como humedad, temperatura y salinidad (Monge, 2008). Asimismo, la distribución de las especies se ajusta a las características del medio físico, el cual es forjado y modificado por factores históricos como la actividad geológica (Cevallos Ferris et al., 2012).

Granados Sánchez et al. (2011) mencionan que el clima y la topografía son los principales factores que crean las características espaciales y temporales de las comunidades vegetales en zonas áridas al limitar la productividad vegetal. Un ejemplo de esto es el levantamiento de cadenas montañosas en el oeste de Norteamérica que alteró la topografía y la distribución de múltiples especies de plantas y animales como las poblaciones de especies del genero Pinus, que en algunos lugares desapareció y en otros, sus poblaciones quedaron como relictos de una distribución histórica más amplia (Sánchez, 2008). En esta revisión se presenta una caracterización biogeográfica de Sierra Rica que aborda los aspectos ecológicos e históricos que moldearon la diversidad vegetal actual y permitirá comprender los patrones de diversidad y distribución de las comunidades vegetales en esta región.

Área de estudio. Sierra Rica ocupa una superficie de 4,464 ha dentro del Área de Protección de Flora y Fauna Cañón de Santa Elena en el municipio de Manuel Benavides, al noreste del estado de Chihuahua, México (Figura 1). La ubicación geográfica es $29^{\circ} 07^{\prime} 39.9^{\prime \prime} \mathrm{N}, 104^{\circ} 10^{\prime} 46.62^{\prime \prime} \mathrm{O}$ y $29^{\circ} 11^{\prime} 36.86^{\prime \prime} \mathrm{N}, 104^{\circ} 04^{\prime} 26.10^{\prime \prime} \mathrm{O}$. La precipitación media anual es de $570.71 \mathrm{~mm}$ y la temperatura media anual oscila entre $18{ }^{\circ} \mathrm{C}$ y $22^{\circ} \mathrm{C}$ (SEMARNAT, 2013). La altitud se encuentra entre los 1,450-2,409 m.s.n.m., la topografía varia de lomeríos de altitud baja hasta cañadas y pendientes escarpadas de más de $30^{\circ}$. El clima según Köppen, modificado por García (2004) es BWhw, y corresponde a árido o desértico (SEMARNAT, 2013). Hidrológicamente, la zona pertenece a la Región Bravo-Conchos RH24 y corresponde a la subcuenca Río Bravo-Arroyo Ventanas; los arroyos principales son La Consolación y Ventanas (INEGI, 2013).

Los suelos presentes son rendizina y litosol. Estos suelos se caracterizan por ser de poca profundidad, descansan sobre rocas ígneas extrusivas y sedimentarias (SEMARNAT, 2012). La vegetación que domina la mayor parte de la zona es bosque de pino; sin embargo, se observa pastizal amacollado arborescente y mediano abierto (COTECOCA, 1978; SEMARNAT, 2013).

Origen geológico. El DC se ha caracterizado y definido por las formaciones orográficas que le rodean: la Sierra Madre Occidental por el oeste, Sierra Madre Oriental por el este y el Eje Neovolcánico Transversal al sur.

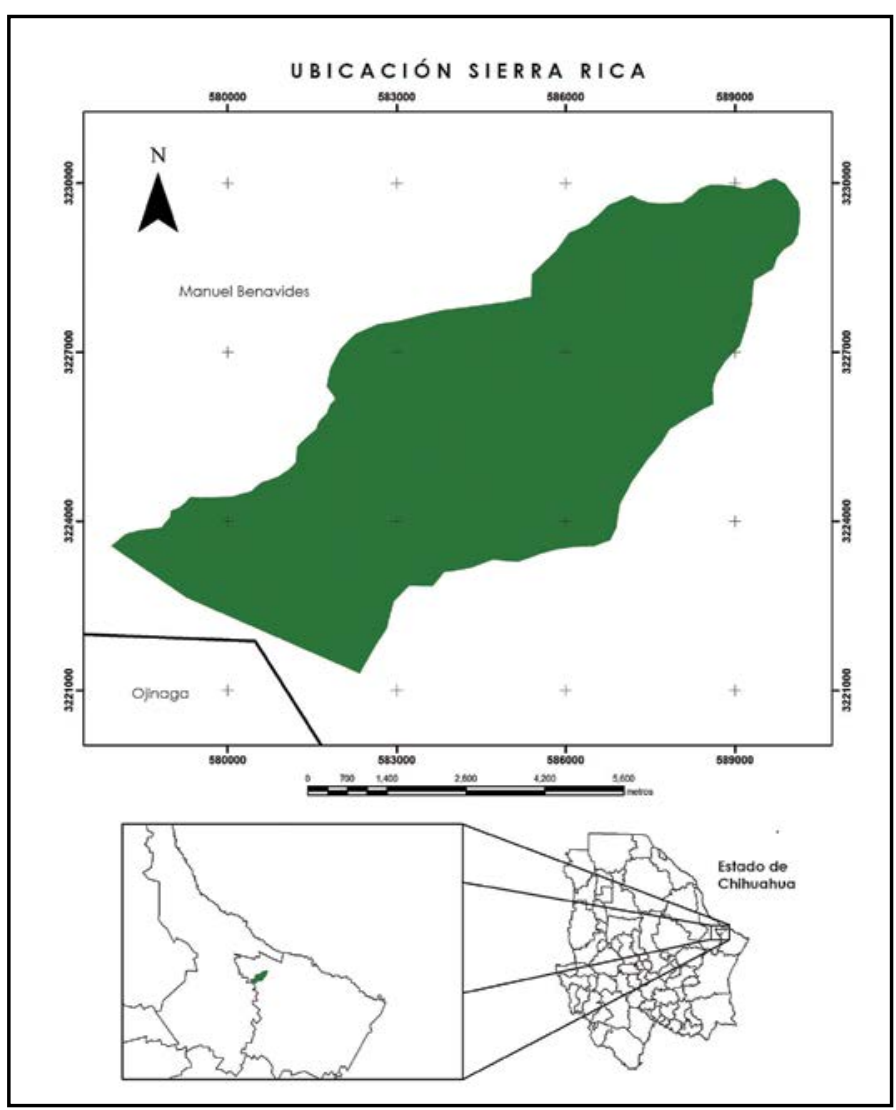

Figura 1. Ubicación de Sierra Rica, Manuel Benavides, Chihuahua, México. Elaboración propia. 
Estas sierras se formaron cuando la planicie mexicana se elevó a través de erupciones volcánicas durante la Era Cenozoica (McClaran y Van Devender, 1995). A este acontecimiento tectónico se le conoce como farallón. Esta actividad generó el choque de las placas de Norteamérica (continental) y la del Pacífico Oriental (oceánica) bajo el continente, lo levantó y originó un intenso vulcanismo (SEMARNAT, 2013).

De manera subsecuente a las fallas tectónicas en el DC se formaron pequeñas islas de montaña. Graham (1999) indica que el origen de las sierras del oeste de Norteamérica fue a través de vulcanismo. Sin embargo, para el este del estado de Chihuahua, SEMARNAP (1997) describe Sierra Rica como una caldera volcánica que se originó en eventos geológicos de la Era Cenozoica.

\section{Factores abióticos determinantes en la distribución de la vegetación}

Clima. En eras geológicas pasadas el DC presentó un clima frio y húmedo con lluvias abundantes en invierno, la vegetación dominante eran árboles de los géneros Pinus y Juniperus (Metcalfe et al., 2000). Sin embargo, los procesos geológicos y los periodos glaciales e interglaciales intervinieron en el cambio de las temperaturas, en la distribución de los organismos y en la formación de barreras fisiográficas en el país. Estas barreras actuaron de manera directa en la distribución de la humedad y temperatura, lo que da lugar a la aridez que caracteriza el DC (Rzedowski, 2006).

Las variables atmosféricas como temperatura, humedad y radiación solar delimitan la distribución de la vegetación de Sierra Rica, estos factores dan lugar a una variabilidad en la composición de especies de diferentes comunidades vegetales. Conforme aumenta la altitud la temperatura disminuye y genera un clima más templado que favorece especies de zonas características de estas regiones (Granados Sánchez et al., 2011).

Las condiciones climáticas tienen un fuerte dominio sobre la distribución geográfica en zonas de transición de bosque a pastizal (D'Odorico et al., 2013). Estas condiciones en conjunto con las características fisiográficas forman microclimas favorables para el establecimiento de diversas formas de vida e incluso para generar especies endémicas con distribución sumamente limitada (Luna Vega et al., 2007). En los cañones se presentan formas de vida distintas a las de mayor altitud, ya que existe mayor humedad debido a que la radiación solar directa es mínima,

lo que generó bosque de galería como el que está presente en el Cañón de los Fresnos en Sierra Rica, con especies como Fraxinus velutina, Prunus serotina y Juglans microcarpa.

Laradiación solarse presenta como unfactorque depende de la altitud, pendiente y exposición. Veera (2005) indica que la misma influye en la distribución de las plantas a través del control que ejerce en la evapotranspiración, así existen diferentes especies en las distintas exposiciones. Esta es una expresión de las diferencias en la evaporación causadas por la radiación solar (Burgess y Northington, 1977). La mezcla de elementos xéricos con elementos templados es característica de Sierra Rica. En exposición sur se observan especies como Aloysia wrigthii, Fouquieria splendens y Yucca carnerosana mezcladas con especies de clima templado como Pinus remota, Prunus serotina y Cercocarpus montanus (Figura 2).

Fisiografía. Las características topográficas, el substrato geológico y el suelo en las regiones áridas son determinantes en la distribución de la vegetación (Rzedowski, 2006). Con base en esto, la distribución de la vegetación de Sierra Rica está determinada principalmente por características fisiográficas debido a que es una zona montañosa de forma lenticular accidentada con un rango altitudinal desde 1,400 hasta 2,409 m.s.n.m., lo que representa un factor de mayor notoriedad y la composición florística cambia en un gradiente altitudinal. González Palma y Sosa Cerecedo (2003) afirman que en esta región el principal factor que determina las comunidades vegetales es la altitud. Poulos y Camp (2005) encontraron que hay una correlación entre la abundancia de árboles y los patrones de distribución con la elevación en Chisos Mountains, en Big Bend National Park, Texas.

La exposición intensifica o disminuye la influencia de otros factores, como la radiación solar y humedad (Good, 1974; Veera, 2005). En Sierra Rica este factor topográfico da lugar a un mosaico de especies que requieren mayor o menor humedad en el suelo. Walton et al. (2005) demostraron que la distribución de la vegetación en la topografía es determinada por la radiación solar.

La fisiografía accidentada de Sierra Rica presenta una serie de cañones y cañadas a diferente rango de altitud con características distintivas de topografía y flora. Los cañones principales son el Cañón de la Madera, Cañón de la Consolación 


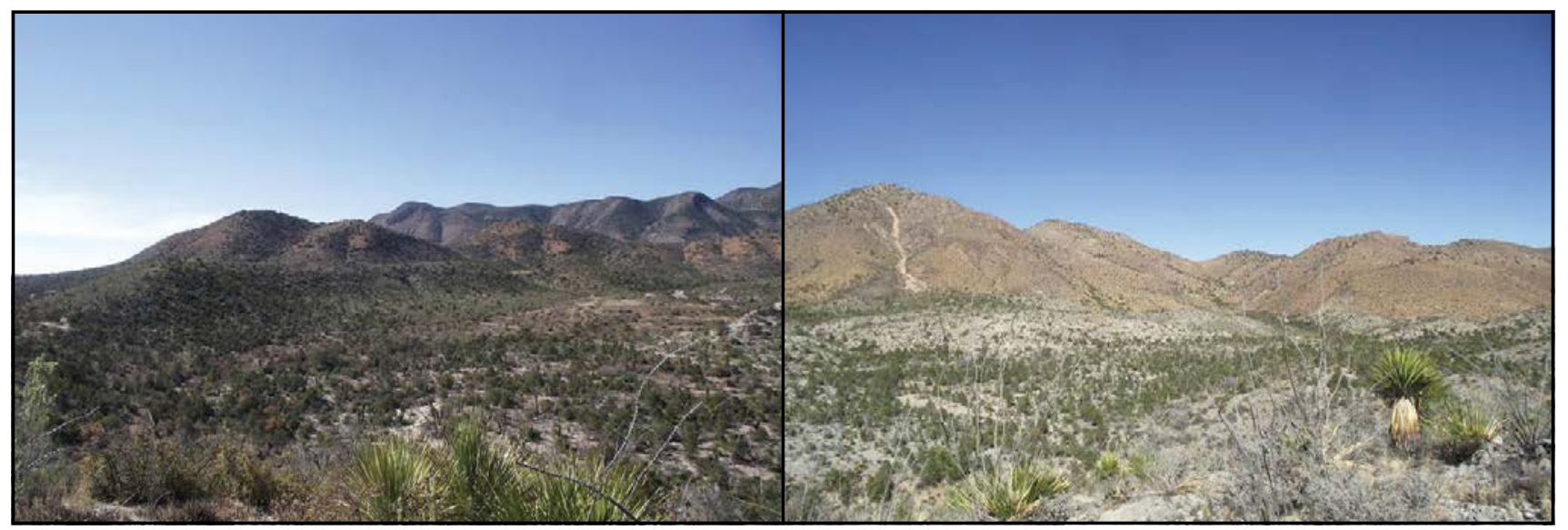

Figura 2. Diferencias de la fisonomía vegetal, exposición norte (izq.) y exposición sur (der.). Sitio ubicado al noreste de la sierra, en Sierra Rica, Manuel Benavides, Chihuahua, México. Fotografía tomada por Alicia Juárez Pérez.

y Cañón de los Fresnos. El Cañón de la Madera se ubica a una mayor elevación, desde los 1,700 hasta los 2,100 m.s.n.m. con especies adaptadas a climas más templados como Cupressus arizonica y Juniperus deppeana. El Cañón de la Consolación se ubica en un rango altitudinal desde los 1,500 hasta los 1,800 m.s.n.m., en donde la vegetación de las partes más bajas son arbóreas como Salix babylonica y Juglans microcarpa, mientras que el Cañón de los Fresnos presenta el mismo rango altitudinal, y diferentes especies como Fraxinus americana, F. velutina y Prunus serotina. Los tres cañones muestran diferentes especies establecidas a distintos rangos de altitud y humedad.

Edafología. El origen volcánico de Sierra Rica da lugar a suelos someros rocosos con diferentes minerales y texturas. Las características físicas y químicas de los suelos son indispensables para el establecimiento de las plantas. Medeiros y Drezner (2012) encontraron que el gradiente del $\mathrm{pH}$ del suelo determina la distribución de las especies Ambrosia deltoidea, A. dumosa y Larrea tridentata.

Los principales suelos de Sierra Rica son leptosoles, en los que la vegetación arbórea y de pastizal son comunes (SEMARNAT, 2013). Se presentan seis unidades edafológicas en el sitio: leptosol calcárico réndzico, leptosol eútrico lítico, leptosol calcárico lítico, leptosol eútrico esquelético y leptosol réndzico esquelético (INEGI, 2015). La unidad edafológica tipo éutrico se presenta como dominante en la zona y la tipo calcárico se presenta en dos áreas (Figura 3), en la parte suroeste y sureste con matorral desértico micrófilo y rosetófilo.

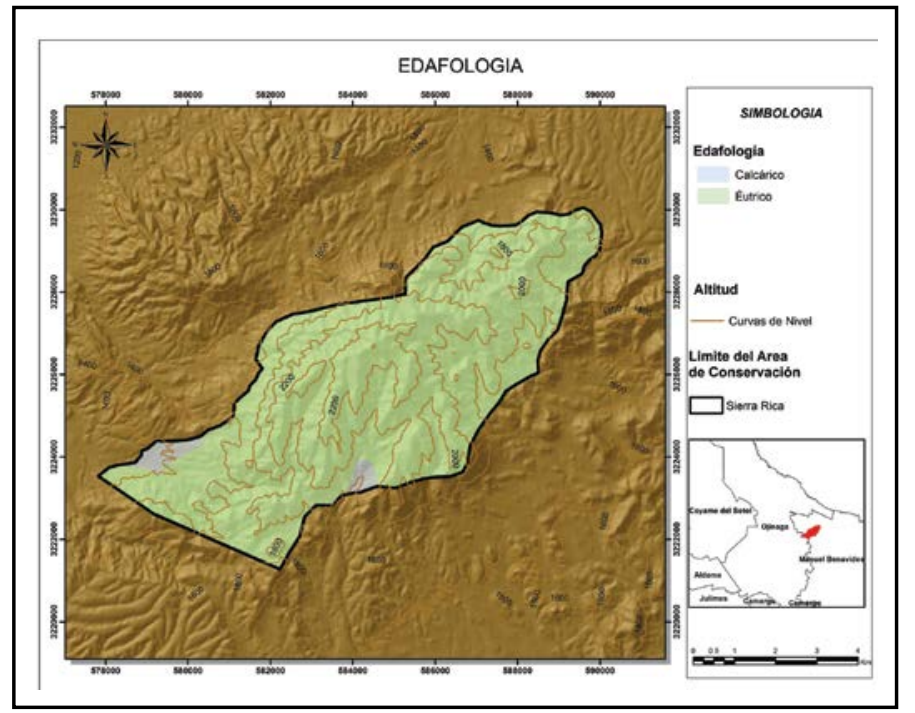

Figura 3. Mapa edafológico de Sierra Rica, Manuel Benavides, Chihuahua, México. Elaboración propia.

\section{Factores bióticos determinantes en la distribución de la vegetación}

Las comunidades vegetales en Sierra Rica presentan una heterogeneidad importante, la cual se debe principalmente a características fisiográficas y climáticas. Sin embargo, existen factores a menor escala que influyen en el establecimiento y distribución de estas comunidades vegetales. Estos factores son las interacciones bióticas, microorganismos del suelo, mecanismos de dispersión y factores evolutivos como flujo y deriva genéticos y presiones de selección.

Las especies actuales son producto de una multitud de factores evolutivos que actúan en conjunto o separados. Factores como la reproducción, filogenia, 


\section{IIVESTIGAGIÓก Y CIECEIA DE LA UNIVERSIDAD AUTÓNOMA DE RGUASCALIERTES}

flujo genético y factores de selección determinan la identidad de las especies (Good, 1974). Las diferentes formas de vida y adaptaciones que presentan las plantas son también el resultado del tiempo evolutivo y el clima; de esta forma, por ejemplo, la adaptación de las plantas a las altas temperaturas por medio de vellosidades en hojas y tallos son producto del proceso evolutivo de millones de años (Rzedowski, 2006).

Wisz et al. (2013) indican que las interacciones bióticas, la dinámica de las especies y su relación con el clima son predictores de la distribución actual y futura de las especies de flora y fauna. Una interacción que se presenta en Sierra Rica, en zonas bajas de matorral desértico y exposiciones de orientación sur, son las plantas nodrizas. Estas trabajan como protectoras de otra especie, esta asociación se observa principalmente en cactáceas (Figura 4).

Otra interacción importante es la existencia del mutualismo entre las micorrizas arbusculares y la flora. Estas existen en todo tipo de ambientes y benefician a la vegetación en el establecimiento y desarrollo de la misma (González et al., 2007); incluso las vuelve más resistentes al estrés hídrico (AI Whaibi, 2009). Las especies perennes cuentan con esta interacción en mayor proporción que las especies anuales, debido a tamaños radiculares y periodos de vida (Collier et al., 2003).

Las plantas utilizan como mecanismos de dispersión las semillas y la reproducción vegetativa. Las semillas son transportadas por medio del viento, el agua y el consumo de estas por los animales. Los mamíferos y las aves emplean mecanismos directos e indirectos para su transporte. Burgess (1995) menciona que los herbívoros son un factor que acelera los cambios en la vegetación y favorecen la variabilidad en las formas de crecimiento que eventualmente generarán poblaciones mejor adaptadas. Los roedores (ratas y ratones), lagomorfos (conejos y liebres) y aves promueven el incremento en la densidad y riqueza del matorral (Whitford y Bestelmeyer, 2006). El jabalí de collar (Pecari tajacu) se alimenta de frutos rojos, granos de gramíneas e insectos, y consume Celtis pallida, Rhus trilobata y R. virens. El venado bura (Odocoileus hemionus) consume los frutos de Echinocereus spp., ampliamente distribuidos en la zona.

Diferentes especies de murciélago del DC, como el murciélago trompudo (Choeronycteris mexicana) y el murciélago magueyero menor (Leptonycteris

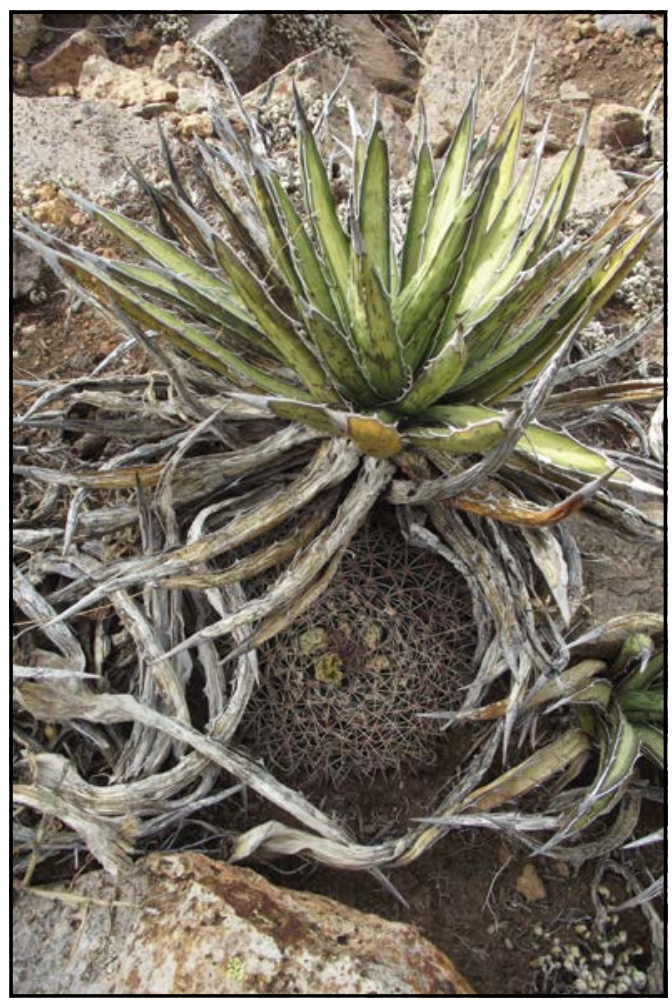

Figura 4. Agave lechuguilla como nodriza de Mammillaria heyderi en Sierra Rica, Manuel Benavides, Chihuahua, México. Fotografía tomada por Alicia Juárez Pérez.

yerbabuenae) han desarrollado una relación de tipo coevolutiva con agaves y cactáceas. Esta interacción se debe a que el murciélago acude a alimentarse a estas plantas y al introducir el hocico y la lengua en la flor se impregna de polen, de esta forma lo dispersa y contribuye a la variabilidad fenética y genética de la planta. Entre los invertebrados, una especie de catarina (Hippodamia convergens) se presentó en cantidades abundantes durante el mes de septiembre, a una altura de 2,409 m.s.n.m. Este insecto es un controlador de plagas natural, se alimenta de áfidos y otros insectos (Marriott, 2012). Esta especie pudiera tener un efecto positivo en la vegetación anual, al protegerla de plagas y usarla como hospedera durante la época de reproducción. En campo, esta especie se observó especialmente sobre plantas anuales.

Otras plantas han desarrollado unareproducción vegetativa como opción para su distribución y reproducción. Tal es el caso de especies de cactáceas como Echinocereus stramineus, Opuntia imbricata y O. leptocaulis, que forman colonias. La gramínea Bouteloua eriopoda con su reproducción 
por estolones puede formar manchones. Un comportamiento similar lo presenta otra gramínea introducida en el área: Cynodon dactylon.

\section{Origen y distribución de las comunidades vegetales}

A través de millones de años se han presentado diferentes fenómenos como el cambio cíclico en las temperaturas y las fallas tectónicas que moldearon los continentes e iniciaron la formación de las distintas comunidades vegetales. En Sierra Rica se distinguen cinco tipos de vegetación principales, como bosque de pino, bosque de encino, pastizal, matorral desértico micrófilo y matorral desértico rosetófilo.

Bosque de pino. El género Pinus es dominante en este tipo de vegetación, del cual se tienen datos de su origen en el periodo Jurásico de la Era Mesozoica (199.6 Ma) en el hemisferio norte (Styles, 1998). La migración de este género hacia el sur comenzó debido a la expansión de las temperaturas frías o templadas generadas por los cambios climáticos y por la fluctuación de las barreras físicas, lo que provocó que llegara a México en el Cenozoico medio (Miller, 1977). Durante el Pleistoceno tardío el bosque de pino dominó el norte del DC con especies de pino piñonero (Pinus spp.), juníperos (Juniperus spp.) y encinos (Quercus spp.) (Lanner y Devender, 1981). Sin embargo, los cambios climáticos y geográficos a través del tiempo fueron fragmentando esta comunidad vegetal hasta quedar como parches en el desierto situados en las islas del cielo. Una característica distintiva en este tipo de vegetación en Sierra Rica es la composición mixta de Yucca spp., con Pinus remota. Existe evidencia de que desde la última glaciación del Pleistoceno estas especies han compartido el mismo espacio, incluso en terrenos más bajos; ahora esta composición se observa en zonas más altas que en el pasado (Wells, 1977).

Bosque de encino. Este tipo de vegetación frecuentemente se asocia al bosque de pino. Sin embargo, hay zonas muy específicas donde este domina en su totalidad. Las evidencias fósiles más antiguas que se tienen del género Quercus datan del Eoceno $(55.8 \mathrm{Ma}$ ) en el norte de Estados Unidos (Manchester, 1983), pero en Texas se encontraron evidencias de hojas de Quercus en la era Cenozoica, periodo Oligoceno (33.9 Ma) con características similares a las especies modernas (Nixon, 1998). Esto sugiere que su origen puede remontarse a épocas anteriores a las que se cree. En el DC están generalmente restringidos a montañas y particularmente a cañones húmedos y laderas de exposición norte y noreste. En Sierra Rica existen cuatro especies: Quercus grisea, Q. vaseyana, Q. pungens y Q. oblongifolia. Se encontraron macrofósiles en áreas bajas de Q. grisea y $Q$. pungens en Big Bend National Park, que datan de la última era glacial (1 1,500 años), a $241 \mathrm{~km}$ al este, lo que sugiere una amplia distribución de estas especies (Wells, 1977; Van Devender 1995).

Pastizal. En el DC, el pastizal se compone de diversos elementos de gramíneas, arbustos y suculentas. La primer comunidad de gramíneas dominantes apareció durante la Era Cenozoica, en el terciario temprano de manera estacional (Van Devender, 1995). Durante el Mioceno ocurrió el levantamiento de cadenas montañosas y las masas de aire del Pacífico fueron bloqueadas por las montañas, creando las condiciones para el desarrollo de las gramíneas en las grandes planicies de Norteamérica (McClaran y Van Devender, 1995). Con estos cambios la vegetación presentó modificaciones y una mayor diversidad de especies, que provocó su expansión hasta las planicies mexicanas.

Matorral. Van Devender (1995) sugiere que el matorral desértico se formó hace 8,980 años. Sin embargo, hay datos sobre especies cuya antigüedad es mayor, como el género Dasylirion, del cual se tienen pruebas de su presencia en la última glaciación hace 10,400 años, así como del género Prosopis, hace 21,300 años. Ambas especies son componentes comunes en matorrales y pastizales.

Conforme se fueron estableciendo las especies arbustivas en los pastizales, más especies llegaban de diversos puntos, como es el caso de Larrea tridentata. Esta especie se expandió a México desde Sudamérica durante el Cuaternario, así como otras especies de los géneros Condalia, Fagonia, Hoffmannseggia, Lycium, Menodora, Nicotiana, Prosopis, Sellaginella y Ziziphus (Rzedowski, 2006).

CONCLUSIONES

En Sierra Rica los factores dominantes en el establecimiento de la flora actual son la fisiografía y el clima. Sierra Rica presenta características que a través de millones de años han formado la heterogeneidad de la vegetación actual. Por su origen volcánico, las especies que iniciaron la colonización fueron Pinus y Quercus. Sin embargo, los acontecimientos tectónicos y climáticos dieron forma al establecimiento de más especies y se redujo la distribución de las especies pioneras. 
IIVESTIGACIÓn Y CIERGIA DE LA UחIVERSIDAD AUTÓnOMH

90 DE RGUASCALIERTES
Las formaciones físicas crean las condiciones de microclima ideales para el establecimiento de flora única (especies endémicas), como el caso de Cupressus arizonica, que solo se ubica en el Cañón de la Madera a una elevación desde 1,800 hasta 2,000 m.s.n.m. Los factores que dan lugar a los tipos de vegetación y su distribución trabajan entre sí, la exposición depende de la radiación solar y la altura para el establecimiento de especies como Dasylirion leiophyllum, Fouquieria splendens y Castilleja indivisa.

El establecimiento de los tipos de vegetación presentes en la zona fue a través de diferentes épocas y sucesos y obedece a factores de tipo ecológico e histórico. Las islas del cielo como Sierra Rica por definición son áreas separadas de otras similares y se considera que han actuado como refugio para múltiples especies (periodos glaciales e interglaciales), que a través del tiempo se han diferenciado de sus poblaciones origen (especiación) y han generado especies endémicas.

En Sierra Rica, al igual que en gran parte del territorio mexicano, la composición y diversidad de especies vegetales y animales está determinada en primer término por la mezcla de elementos provenientes de las dos regiones biogeográficas del continente (la Neártica y la Neotropical) y por un elemento endémico, que se ha desarrollado debido a las condiciones fisiográficas y a las fluctuaciones climáticas del Pleistoceno.

En la actualidad los cambios en la estructura y distribución de la vegetación continúan. Sin embargo, existen amenazas antropogénicas que modifican esta estructura, como el sobrepastoreo y la introducción de especies exóticas, como Melinis repens, Cynodon dactylon y Salsola iberica.

\section{LITERATURA CITADA}

- $\quad A L$ WHAIBI, M. H. Desert plants and Mycorrhizae (A mini-review). Journal of Pure and Applied Microbiology, 3(2): 457-466, 2009.

- BURGESS, T. L. Desert grassland, mixed shrub savanna, shrub steppe, or semidesert scrub? The dilemma of coexisting growth forms. En M. P. McCLARAN y T. R. VAN DEVENDER (Eds.), The Desert Grassland (31-67). Arizona: University of Arizona Press, 1995.

- BURGESS, T. L. y NORTHINGTON, D. K. Desert vegetation in the Guadalupe Mountains region. En R. H. WAUER y D. H. RISKIND (Eds.), Transactions of the Symposium on the Biological Resources of the Chihuahuan Desert Region (229-242). Washington, US: US Department of the Interior, National Park Service, 1977.

- CEVAlLOS FERRIZ, S. R. S. et al. Perspectiva paleobotánica y geológica de la biodiversidad en México. Acta Botánica Mexicana, 100, 317-350, 2012.

- COLLIER, S. C. et al. Mycorrhizal dependency of chihuahuan desert plants is influenced by life strategy and root morphology. Journal of Arid Environments, 55(2): 223-229, 2003.

- CONABIO (COMISIÓN NACIONAL PARA El CONOCIMIENTO Y USO DE LA BIODIVERSIDAD). La biodiversidad en Chihuahua: Estudio de estado. D. F., México: Autor, 2014.

- cotecoca lCOMISIÓn técnico consultiva para LA DETERMINACIÓN REGIONAL DE LOS COEFICIENTES DE AGOSTADERO). Estudio para el Estado de Chihuahua. D. F., México: Secretaría de Agricultura y Recursos Hidráulicos, 1978.
- COX, C. B. y MOORE, P. D. Biogeography, an ecological and evolutionary approach. 5 ed. Londres, Gran Bretaña: Blackwell Scientific Publications, 336 pp., 1993.

- D'ODORICO, P. et al. Vegetation-microclimate feedbacks in woodland-grassland ecotones. Global Ecology and Biogeography, 22 (4): 364-379, 2013.

- GARCÍA, E. Modificaciones al sistema de clasificación climática de Köppen. D. F., México: Universidad Nacional Autónoma de México, 2004.

- GONZÁlez CHÁVEZ, M. C. et al. Biodiversidad funcional de los hongos micorrícicos arbusculares en zonas áridas y semiáridas. En N. M. MONTAÑO ARIAS et al. (Eds.), Micorrizas arbusculares en ecosistemas áridos y semiáridos (37-62). D. F., México: Instituto Nacional de Ecología/SEMARNAT-Mundi-Prensa, S. A. de C. V.-UAM Iztapalapa-FES Zaragoza-UNAM, 2007.

- GONZÁlez, PALMA, A. y SOSA CERECEDO, M. Análisis de la vegetación del área de protección de flora y fauna Cañón de Santa Elena (desierto chihuahuense) utilizando Modelos Digitales de Elevación. Ecosistemas, 12(2): 1-6, 2003.

- GOOD, R. The geography of the flowering plants. 4 ed. United Kingdom: Longman Group, 403 pp., 1974.

- Graham, A. Late Cretaceous and Cenozoic History of North American vegetation. North of Mexico. New York, US: Oxford University Press, 350 pp., 1999.

- GRANADOS SÁNCHEZ, D. et al. Ecología de la vegetación del desierto chihuahuense. Rchscfa (Revista Chapingo Serie Ciencias Forestales y del Ambiente), XVII, 111-130, 2011. 
- INEGI (INSTITUTO NACIONAL DE ESTAdístiCA y GEOGRAFíA). Anuario estadístico y geográfico por entidad federativa. México: Autor, 744 pp., 2013.

- LANNER, M. R. y VAN DEVENDER, T. R. Late Pleistocene pines in the chihuahuan desert. Quaternary Research, 15(3): 278-290, 1981.

- LOMOLINO, M. V. et al. Biogeography. 4 ed. Sinaver Associates Inc., 764 pp., 2010.

- LUNA VEGA, I. et al. (Eds.), Biodiversidad de la Faja Volcánica Transmexicana. D. F., México: Comisión Nacional para la Biodiversidad-Universidad Nacional Autónoma de México, 2007.

- MANCHESTER, S. R. Eocene fruits, wood and leaves of the Fagaceae from the clarno formation of Oregon. American Journal of Botany, 70(5 PART 2): 70-74, 1983.

- MARRIOTT, S. M. Assessing Geographic Distribution and Relative Abundance Patterns of Native and Non-Native Lady Beetles (Coccinellinae) from Historic Occurrence Data. Tesis de Maestría. Canada: Facultad de Ciencias, Universidad de Prince Edward Island, 2012.

- McClaran, M. P. y VAN DEVENDER, T. R. The Desert Grassland. Tucson, Arizona, US: University of Arizona Press, 346 pp., 1995.

- MCCORMACK, J. E. et al. Sky islands. En R. G. GILLESPIE y D. CLAGUE (Eds.), Encyclopedia of Islands (838-843). Berkeley, California: University of California Press, 2009.

- MEDEIROS, A. S. y DREZNER, T. D. Vegetation, climate, and soil relationships across the sonoran desert. Ecoscience, 19(2): 148160, 2012

- METCALFE, S. E. et al. Records of Late Pleistocene-Holeocene climatic change in Mexico-a review. Quaternary Science Reviews, 19(7): 699-721, 2000.

- MILLER, C. N. Mesozoic conifers. The Botanical Review, 43, 217280, 1977.

- MONGE NÁJERA, J. Ecological biogeography: a review with emphasis on conservation and the neutral model. Gayana, 72(1): 102-112, 2008.

- NIXON, K. C. El género Quercus en México. En T. P. RAMAMOORTHY et al. (Comps.), Diversidad Biológica de México. Orígenes y distribución (447-458). D. F., México: Universidad Nacional Autónoma de México, 1998.

- POULOS, M. H. y CAMP, A. E. Vegetation-environment relations of the Chisos Mountains, Big Bend National Park, Texas. USDA Forest Service Proceedings RMRS-P-36, 539-544, 2005.

- SÁNCHEZ GONZÁLEZ, A. Una visión actual de la diversidad y distribución de los pinos de México. Madera y Bosques, 14(1): 107-120, 2008.
- SEMARNAP ISECRETARÍA DE MEDIO AMBIENTE, RECURSOS NATURALES Y PESCA). Programa de Manejo Área de Protección de Flora y Fauna Cañón de Santa Elena. D. F., México: Instituto Nacional de Ecología, 134 pp., 1997.

- SEMARNAT (SECRETARÍA DE MEDIO AMBIENTE y RECURSOS NATURALES). Atlas de Medio Físico Biótico y Ambiental del Estado de Chihuahua. D. F., México: Autor, 2012.

- SEMARNAT (SECRETARÍA DE MEDIO AMBIENTE Y RECURSOS NATURALES)-CONANP (COMISIÓN NACIONAL DE ÁREAS NATURALES PROTEGIDAS). Programa de Manejo Área de Protección de Flora y Fauna Cañón de Santa Elena. D. F. México: CONANP, 153 pp., 2013.

- STYles, T. B. El género Pinus: su panorama en México. En T. P. RAMAMOORTHY et al. (Eds.), Diversidad biológica de México, orígenes y distribución (397-420). D. F., México: Universidad Nacional Autónoma de México, 1998.

- VAN DEVEnder, T. R. Desert Grassland History. En M. P. MCCLARAN y T. R. VAN DEVENDER (Eds.), The Desert Grassland. Tucson, AZ, US: University of Arizona Press, 1995.

- VEeRA, V. M. Hydrology and Solar Radiation as Factors for desert Vegetation Patterns. Tesis de Maestría. El Paso, TX, US: Universidad de Texas, 2005.

- WALTON, J. C. et al. Desert vegetation and timing of solar radiation. Journal of Arid Environments, 60(4): 697-707, 2005.

- WELLS, P. V. Post-glacial origin of the present Chihuahuan Desert less than 11,500 years ago. En R. H. WAUER y D. H. RISKIND (Eds.), Transactions of the Symposium on the Biological Resources of the Chihuahuan Desert Region, United States and Mexico (6783). Alpine, TX, US: Sul Ross State University, 1977.

- WhITFORD, W. G. y BESTELMEYER, B. Chihuahuan desert fauna: effects on ecosystem properties and processes. En F. M. HAVSTAD et al. (Eds.), Structure and Function of a Chihuahuan Desert Ecosystem: The Jornada Basin Long-Term Ecological Research Site (247-265). New York, NY, US: Oxford University Press, 2006.

- WISZ, M. S. et al. The role of biotic interactions in shaping distributions and realized assemblages of species: implications for species distribution modelling. Biological Reviews, 88(1): 1530, 2013.

\section{De página electrónicas}

- INEGI (INSTITUTO NACIONAL DE ESTAdísticA y GEOGRAFía). Edafología, Conjunto de Datos vectorial Edafológico escala 1:250 000 Serie II (Continuo Nacional). Recuperado el 2 de marzo de 2015, de http://www.inegi.org.mx/geo/contenidos/ recnat/edafologia/vectorial_serieii.aspx

- RZEDOWSKI, J. Vegetación de México. Edición digital. D. F. México: Comisión Nacional para el Conocimiento y Uso de la Biodiversidad, 2006. Recuperado de http://www.biodiversidad. gob.mx/publicaciones/librosDig/pdf/VegetacionMxPort.pdf 